\title{
Competitividad electoral y formación de mayorías en El Salvador (1982-1997)
}

\section{Introducción}

Dentro de la literatura politológica existe un inacabado debate en tomo a las "leyes de Duverger" sobre la relación entre sistemas electorales y sistemas de partidos. Duverger (1974) planteó que los sistemas de mayoría absoluta y los sistemas proporcionales están asociados con sistemas multipartidistas. El cúmulo de investigaciones pertinentes gira en tomo a los efectos de los sistemas de representación proporcional sobre el número de partidos. Este resultado es lógico si se considera que la base empírica de tales trabajos suele referirse a sistemas parlamentarios.

El análisis que sigue se refiere a la otra parte de la relación. Pero no lo voy a realizar en términos causales de uno (el sistema mayoritario) sobre el otro (el sistema de partidos). Más bien voy a incursionar en sus efectos conjuntos sobre la necesidad del ballotage que provocan para legitimar al triunfador. Con este objetivo, examino primero los niveles de competitividad y margen de victoria que se han registrado en El Salvador desde 1982. Luego, identifico el formato partidista de la competencia para entonces pasar al análisis de la formación de mayorías en contextos de baja competitividad y multipartidismo. Algunas de las afirmaciones hechas las respaldo con evidencia empírica proveniente de la comparación con lo que sucede en,otros países centroamericanos. Como resultado de este breve trabajo, cae bajo cuestionamiento el criterio normado para definir al partido ganador en las elecciones presidenciales salvadoreñas.

\section{Competitividad electoral y margen de victoria (1982-1997)}

Referirse a la competitividad de las elecciones no es 10 mismo que hablar de elecciones competitivas. Estas últimas constituyen un tipo ideal frente al cual se comparan las elecciones reales. En este sentido, además de elecciones competiti- 
vas también se habla de elecciones semicompetitivas y no competitivas. Los criterios centrales para definir esta tipología tienen que ver con: la oportunidad de elegir entre differentes opciones y la libertad de elección (Krennerich, 1993). La limitación de éstas o su prohibición convierten a las elecciones competitivas en semicompetitivas o no competitivas respectivamente.

Ahora bien, la competitividad es una característica de, y no un tipo de, las elecciones. Obviamente, si no hay oportunidad de elección ni libertad para hacerlo, la competitividad es nula. Es el caso de las elecciones no competitivas. Sin embargo, tanto en las elecciones competitivas como en las semicompetitivas se pueden presentar diversos grados de competitividad según, lo que llamaré de aquí en adelante, el margen de victoria. Éste consiste en la diferencia en las proporciones (normalmente expresada en puntos porcentuales) de votos o escaños entre los dos primeros partidos en cada elección ${ }^{2}$. Por asociación diremos que cuanto mayor sea el margen de victoria, menor es la competitividad de las elecciones y viceversa. Además, si lo que se toma en cuenta son los votos, hablaremos entonces de margen de victoria electoral. Si lo que se considera son los escaños (en elecciones parlamentarias), entonces hablaremos de margen de victoria parlamentaria.

Como el margen de victoria puede ser grande aun en sistemas competitivos, de allí se deduce que la competitividad (asociada al primero) no es un criterio para discemir el tipo de elección (si es competitiva o semicompetitiva), pero puede ser utilizado para analizar el comportamiento del electorado, a niveles agregados, en distintos períodos. De particular interés puede resultar el análisis de los períodos de cambio de régimen político para identificar continuidades y cambios en dicho comportamiento.

El Cuadro 1 muestra los distintos valores que asume el margen de victoria electoral, para el caso de las elecciones presidenciales salvadoreñas entre 1984 y 1994. El Cuadro 2 se refiere a las elecciones parlamentarias entre 1982 y 1997. En este último cuadro agrego también el margen de victoria parlamentario. Al calcular los promedios para todo el período se obtiene 18.5 para las elecciones presidenciales; 14.8 y 15.2 para las parlamentarias. Todos estos valores sugieren (por asociación) bajos niveles de competitividad; lo cual puede interpretarse como la posibilidad de conocer el resultado de antemano sobre todo cuando se cuenta con resultados provenientes de encuestas de opinión. Esta posibilidad supone menor nivel de incertidumbre respecto del resultado electoral, al menos en términos del probable vencedor. Otra cosa es que, por un diseño institucional, deba decidirse tal resultado con una segunda vuelta entre los dos primeros partidos. Aquí se plantea el problema del criterio para decidir sobre la necesidad de una segunda vuelta cuando el margen de victoria, en primera vuelta, es tal que simplemente se confimará el resultado ya obtenido. Lo sucedido en 1994 debería ser materia de reflexión para el Tribunal Supremo Electoral. Por un escaso 1 
por cient de votos fue necesaria una segunda vuelta entre ARENA y la Coalición FMLN-CD-MNR. El margen de victoria, en primera vuelta, fue de 24 puntos porcentuales. La segunda vuelta confirmó (legitimó ampliamente) el triunfo arenero pero también significó un mayor desgaste procedimental y, sobre todo, financiero. Aquella distancia electoral difícilmente iba a ser superada (revertida) en la segunda vuelta.

\section{Cuadro 1}

Margen de victoria en las elecciones presidenciales entre 1984 y 1994 (en porcentajes)

\begin{tabular}{rcl}
\hline Año & Margen de victoria & \multicolumn{1}{c}{ Partidos o coaliciones } \\
\hline 1984 & $13.6^{*}$ & PDC - ARENA \\
1989 & 17.8 & ARENA - PDC \\
1994 & $24.1^{*}$ & ARENA - FMLN/CD/MNR \\
\hline
\end{tabular}

- Sólo primera vuelta.

Fuente: elaboración propia con base en los resultados oficiales.

\section{Cuadro 2}

Margen de victoria en las elecciones parlamentarias entre 1982 y 1997 (en porcentajes)

\begin{tabular}{c|cc|c}
\hline \multirow{2}{*}{ Año } & \multicolumn{2}{|c|}{ Margen de victoria } & \multirow{2}{*}{ Partidos } \\
\cline { 2 - 3 } & Electoral & Parlamentaria & \\
\hline \multirow{2}{*}{1982} & 10.6 & 8.3 & PDC - ARENA \\
1985 & 22.6 & 33.4 & PDC - ARENA \\
1988 & 12.8 & 11.7 & ARENA - PDC \\
1991 & 16.3 & 15.4 & ARENA - PDC \\
1994 & 23.6 & 21.4 & ARENA - FMLN \\
1997 & 2.4 & 1.2 & ARENA - FMLN \\
\hline
\end{tabular}

Fuente: elaboración propia con base en los resultados oficiales.

No estoy planteando que se suprima la segunda vuelta, sino que se revise el criterio exigido para que ésta tenga que llevarse a cabo. Si atendemos a lo que ocurrió en 1984, aunque la competitividad fue mayor, dado el menor margen de victoria, los 13.6 puntos porcentuales de diferencia entre el Partido Demócrata Cristiano (PDC) y ARENA en la primera vuelta tampoco fueron revertidos en la segunda vuelta. Si el PDC ganó en la primera, también lo hizo en la segunda. En 
El Salvador no se ha dado el caso en el que el ganador de la primera vuelta pierda en la segunda ronda.

En el ámbitołde la ciencia política, se reconoce que un sistema electoral basado en la mayoría absoluta, a dos vueltas, tiene por finalidad la creación artificial de mayorías para legitimar ampliamente el mandato presidencial. Se obliga al electorado a escoger entre los dos candidatos más votados en la primera vuelta. A esta opción institucional se le denomina ballotage (Nohlen, 1994). Con el objetivo de mostrar que realmente se trata de una opción institucional (entre otras), el Cuadro 3 se refiere a lo normado en Centroamérica, pues, como dice el dicho: "como muestra basta un botón".

\section{Cuadro 3}

Mayoría exigida en las elecciones presidenciales de los países centroamericanos

\begin{tabular}{llll}
\hline País & Año & \multicolumn{1}{c}{ Mayoría } & Ballotage \\
\hline Guatemala & 1995 & Absoluta & Sí \\
El Salvador & 1994 & Absoluta & Sí \\
Honduras & 1997 & Simple & No \\
Nicaragua & 1996 & $>45 \%$ de votos válidos & Sí \\
Costa Rica & 1998 & $>40 \%$ de votos válidos & Sí \\
\hline
\end{tabular}

Fuente: elaboración propia según legislación electoral de cada país.

Por otra parte, el margen de victoria registrado en 1997 , y por tanto el nivel de competitividad ese mismo año, puede sugerir un cambio en el comportamiento electoral entre el período de transición 1982-1994 y el período que actualmente vive el país. Aunque ya en 1989 hubo altemancia en el gobiemo; a juzgar por la competitividad de 1997, las elecciones de 1999 podrían presentar una mayor incertidumbre respecto al resultado final. Una situación así es saludable para el juego democrático, porque los principales partidos pueden verse obligados a orientarse centrípetamente reduciendo así los niveles de polarización característicos del período anterior. Sin embargo, las anteriores afirmaciones permanecen en un nivel hipotético sin afán predictivo. Los resultados de las elecciones de 1999 darán más elementos de análisis para trabajar sobre la argumentación acá sostenida y plantearse seriamente la revisión de la correspondiente parte de nuestra legislación electoral. El ballotage podría ser exigido ya no según la mayoría absoluta, sino, como en Nicaragua y Costa Rica, entre el 40 y 45 por ciento de los votos válidos, o bien según la competitividad electoral. 
Cuadro 4

Centroamérica: margen de victoria electoral y parlamentaria

(1980-1998) (porcentajes promedios)

\begin{tabular}{l|cccc}
\hline \multirow{2}{*}{ País } & \multicolumn{4}{|c}{ Margen de victoria } \\
\cline { 2 - 5 } & Electoral & Parlamentaria & Máxima & Mínima \\
\hline Guatemala & 10.2 & 19.6 & $14.5(1981)$ & $5.1(1990)$ \\
El Salvador & 14.8 & 15.2 & $23.6(1994)$ & $2.4(1997)$ \\
Honduras & 8.6 & 9.2 & $12.3(1981)$ & $5.5(1985)$ \\
Nicaragua & 24.9 & 23.3 & $52.8(1984)$ & $8.8(1996)$ \\
Costa Rica & 9.5 & 10.5 & $26.1(1982)$ & $5.2(1994)$ \\
\hline
\end{tabular}

Fuente: claboración propia con base en los resultados de las elecciones parlamentarias.

Con el objetivo de tener una perspectiva comparada, el Cuadro 4 muestra los valores del margen de victoria electoral y parlamentaria en los países centroamericanos entre 1980 y 1997. Como puede observarse, en términos medios Honduras y Costa Rica tienen los sistemas más competitivos con márgenes de victoria prácticamente por debajo de los diez puntos. Nicaragua está en el otro extremo, superando los 20 puntos porcentuales. El Salvador se ubica en una posición intermedia.

\section{Los competidores relevantes}

Otra manera de estudiar la competitividad de las elecciones viene dada por el análisis de los partidos que marcan la pauta de la competencia. Puede decirse que cuanto mayor sea el margen de victoria, menor peso tienel. los partidos ubicados desde la tercera posición hacia abajo. Sin embargo, hay dos indicadores que permiten apreciar mejor el peso de los partidos en la competencia. Por un lado está la concentración del voto (o de los escaños) en los dos primeros partidos y, por otro lado, lo que Sartori (1992) llama formato del sistema de partidos.

El Cuadro 5 muestra la concentración del voto en las elecciones presidenciales salvadoreñas del período 1984-1994. En términos medios, los dos primeros partidos obtuvieron el 79 por ciento de los votos, dejando apenas un 21 por ciento para el resto de partidos. Cuando fue necesaria la doble vuelta (1984 y 1994), los dos primeros partidos concentraron cerca del 74 por ciento de los votos. Cuando no se necesitó la segunda vuelta (1989), la concentración fue casi del 90 por ciento. 


\section{Cuadro 5}

Concentración del voto en los dos primeros partidos

, en elecciones presidenciales (1984-1994)

(en porcentajes)

\begin{tabular}{ccc}
\hline Año & Concentración & Partidos o coaliciones \\
\hline 1984 & 73.2 & PDC y ARENA \\
1989 & 89.8 & ARENA y PDC \\
1994 & 74.1 & ARENA y FMLN-CD-MNR \\
\hline Promedio & 79.0 & \\
\hline
\end{tabular}

Fuente: elaboración propia con base en los resultados electorales.

Si en lugar de las elecciones presidenciales consideramos las parlamentarias, la concentración del voto registra los valores mostrados en el Cuadro 6. En promedio, la concentración es menor que en el caso del Cuadro 5, aunque la diferencia es de poco más de 5 puntos porcentuales. Este detalle es interesante porque a pesar de que el sistema proporcional empleado en las elecciones parlamentarias podría "premiar" a más partidos (por cuanto el primer partido no se "lleva todo", como en los sistemas mayoritarios), el predominio electoral de los dos primeros en las elecciones presidenciales no se ve reducido significativamente en las elecciones parlamentarias.

\section{Cuadro 6}

Concentración del voto y escaños en los dos primeros partidos en elecciones parlamentarias (1982-1997)

(en porcentajes)

\begin{tabular}{l|cc|c}
\hline \multirow{2}{*}{ Año } & \multicolumn{2}{|c|}{ Concentración } & \\
\cline { 2 - 3 } & Voto & Escaños & Partidos \\
\hline 1982 & 69.8 & 71.7 & PDC - ARENA \\
1985 & 82.0 & 76.6 & PDC - ARENA \\
1988 & 83.2 & 88.3 & ARENA - PDC \\
1991 & 72.3 & 77.4 & ARENA - PDC \\
1994 & 66.4 & 67.8 & ARENA - FMLN \\
1997 & 68.4 & 65.4 & ARENA - FMLN \\
\hline Promedio & 73.7 & 74.5 & \\
\hline
\end{tabular}

Fuente: elaboración propia. 
Este predominio electoral y parlamentario de dos partidos no debe ser confundido con un esquema bipartidista. Para sostener empíricamente esta afirmación resulta útil el análisis del formato del sistema de partidos, es decir, el análisis del número de partidos que son relevantes en términos competitivos. La determinación de este número es una cuestión que ha sido ampliamente debatida en los círculos académicos de la ciencia política. Según Lijphart (1987, p. 131): "el problema más importante para determinar la cantidad de partidos que hay en el sistema político radica en si se tiene o no en cuenta a los partidos pequeños, y en todo caso, en el volumen que debe tener un partido para ser contabilizado". El problema radica, pues, en si debe tomarse o no en cuenta a todos los partidos que participan en la contienda electoral. Sartori (1992, pp. 154-157) propuso las siguientes normas para contar:

(a) Considerar sólo los partidos que alcanzan escaños.

(b) No contar aquellos que, aun estando presentes en el parlamento, no han participado en una mayoría de coalición. Es decir, contar los partidos que tienen un potencial para formar una coalición mayoritaria.

(c) Contar a los partidos antisistema que, aunque no hayan formado una mayoría de coalición, tienen una capacidad de veto como para alterar la dirección de la competencia, es decir, contar a los partidos con potencial de chantaje.

A estas reglas para contar se les critica su origen. Están basadas en los sistemas parlamentarios quedando fuera el efecto de las elecciones presidenciales en los sistemas presidencialistas. Por otra parte, ya antes que Sartori, Rae (1967) propuso utilizar el índice de fraccionalización $(F)$ como medida del número de partidos. Dicho índice está basado en la fuerza o peso de cada partido medido en términos de votos o escaños ${ }^{3}$. Una mayor fraccionalización implicará un mayor número de partidos. Los valores de $F$ varían entre 0 y 1 , correspondiendo el primero al caso de un "sistema" de partido único, y el último, a un sistema donde habrían tantos partidos como escaños. Un valor de $F$ de 0.5 corresponde al caso del bipartidismo, y un valor de $F$ de 0.67 indica un sistema de tres partidos.

Laakso y Taagepera (1979) desarrollaron un índice para calcular directamente el número efectivo de partidos $(N)$. Este nos dice el número de partidos hipotéticos del mismo tamàno, que tendrían el mismo efecto sobre la fraccionalización del sistema que producen los partidos realmente existentes ${ }^{4}$. El índice de Laakso y Taagepera $(N)$ comporta la misma información que el índice de Rae $(F)$. De hecho, uno puede calcularse a partir del otro ${ }^{5}$. Sin embargo, $N$ tiene la ventaja de ser más fácilmente visualizado que $F$ como el número de partidos. Por ejemplo, en un bipartidismo con partidos igualmente fuertes, $N$ es exactamente 2 mientras que $F$ es 0.5 . Si uno de los dos partidos es más fuerte 
que el otro, con proporciones de votos o escaños de, por ejemplo, 70 y 30 por ciento, entonces $N$ es 1.72 , lo que indica que el sistema se mueve hacia el predominio de uno de los dos partidos o, por lo menos, que se aleja de la situación de bipartidismo puro (Lijphart, 1994).

El Cuadro 7 muestra la evolución del número efectivo de partidos, tanto electorales (Nv) como parlamentarios (Ns) en el período 1982-1997. He optado por $N$ frente a las normas de Sartori por su fácil interpretación y su aplicabilidad, tanto en las elecciones parlamentarias como en las elecciones presidenciales. Como puede verse en el mencionado cuadro, lejos de estar en una situación de bipartidismo, en El Salvador la competencia electoral se estructura alrededor de un poco más de tres partidos. La afirmación anterior puede parecer una perogrullada para el análisis local, pero no lo es desde el punto de vista de la política comparada ${ }^{h}$. En términos medios, $N$, para el caso de las elecciones presidenciales, ha resultado de 2.9. ¿Qué implicaciones tiene este formato para la competitividad electoral?

Evolución del número efectivo de partidos
en El Salvador (1982-1997)

\section{La formación de mayorías en El Salvador}

Dado el carácter fluido del sistema de partidos salvadoreño', en lugar de asociar partidos políticos específicos con $N$, resulta más adecuado referirse a polos electorales. Al momento de escribir este trabajo, dos de esos polos coinci- 
den con dos partidos políticos: ARENA y el FMLN. El tercer polo electoral queda definido respecto a estos dos partidos. Se trata de los electores que optan por otros partidos, sin que ninguno de estos constituya desafío alguno para las aspiraciones y el predominio de los dos primeros. Sin embargo, un valor de $\mathbf{N}$ de 2.9 implica la no existencia de bipartidismo. Por tanto, el tercer polo electoral se convierte en "fiel de la balanza" en la disputa presidencial entre los dos partidos más fuertes. Opera aquí una especie de "ingeniería electoral" para manufacturar la mayoría absoluta exigida para llevarse la presidencia. Tanto en 1984 como en 1994 operó este mecanismo. En 1989, los dos primeros partidos (ARENA y el Partido Demócrata Cristiano) concentraron casi el 90 por ciento de los votos y no hubo necesidad de una segunda vuelta. Con estos niveles de concentración del voto es difícil que se tenga que realizar una segunda vuelta. Al menos es lo que sugiere el Cuadro 8, que se refiere a Centroamérica en los años comprendidos entre 1980 y 1998. Cuando los niveles de concentración del voto están por debajo del 80 por ciento, como en Guatemala y El Salvador, la doble vuelta es prácticamente ineludible en formatos de 3 o más partidos. En estos casos, el electorado del tercer partido resulta crucial para el resultado final.

\section{Cuadro 8}

Concentración del voto en elecciones presidenciales centroamericanas (1980-1998) (en porcentajes)*

\begin{tabular}{lccccc}
\hline Año & Guatemala & El Salvador & Honduras & Nicaragua & Costa Rica \\
\hline 1981 & - & - & 95.5 & - & - \\
1982 & - & - & - & - & 92.4 \\
1984 & - & 73.2 & - & 81.0 & - \\
1985 & 58.8 & - & 96.5 & - & - \\
1986 & - & - & - & - & 98.1 \\
1989 & - & 89.8 & 96.6 & - & - \\
1990 & 49.8 & - & - & 95.5 & 98.7 \\
1993 & - & - & 96.6 & - & - \\
1994 & - & 74.1 & - & - & 97.3 \\
1995 & 58.5 & - & - & - & - \\
1996 & - & - & - & 88.0 & - \\
1997 & - & - & 95.4 & - & - \\
1998 & - & - & - & - & 91.6 \\
\hline
\end{tabular}

* Cifras referidas a los dos primeros partidos o coaliciones.

Fuente: elaboración propia. 
Un breve recorrido por la historia político-electoral salvadoreña proporciona una mayor sustentación empírica a la relación entre sistema electoral de mayoría absoluta y formato del sistema de partidos. No me interesa en este momento algún tipo de causalidad entre estas dos variables, en el sentido de las largamente debatidas "leyes de Duverger" (Rial, 1988). Voy a centrarme más bien en sus mutuos efectos sobre la necesidad o no del ballotage y en el condicionamiento que ejercen sobre las posibilidades de alternancia en el gobiemo. Respecto de este último punto puedo decir que, en El Salvador, la alternancia en el gobierno sólo ha sido posible cuando, o bien el formato tiende a 2, o bien la "oposición" se aglutina en un único polo electoral. Mi intención acá no es establecer una ley politológica, sino mostrar cómo al manufacturar una mayoría, cuando el formato del sistema de partidos tiende o supera al 3, lo que puede discutirse es el criterio empleado para recurrir al ballotage. Como ya mencioné anteriormente, el resultado de éste sólo ha confirmado (legitimado) al vencedor de la primera vuelta.

\section{1 . La competencia electoral antes de 1982}

En 1824, a escasos tres años de lograda su independencia política de España, entró en vigor la primera Constitución Política salvadoreña que preveía una separación entre un Ejecutivo (liderado por un jefe de Estado) y un Legislativo (basado en un Congreso unicameral). Sin embargo, la lucha por el poder entre liberales y conservadores se resolvió básicamente de forma militar hasta 1871 , año en que entró en vigencia una nueva Constitución de corte liberal, que seguida de otras cuatro en un período de 15 años pretendían consolidar un estado oligárquico liberal. A partir de entonces, las luchas entre liberales y conservadores perdieron importancia superándose temporalmente el mecanismo del golpe de Estado para el cambio de gobiemo.

Entre 1903 y 1931, casi todos los cambios se produjeron, al menos formalmente, por medio de elecciones, aunque poco competitivas, pues los candidatos provenían de unas pocas familias ligadas a la oligarquía cafetalera (Krennerich, 1992, p. 307). Desde 1932 hasta 1979, el control del Ejecutivo pasó a manos de los militares y la sucesión presidencial combinó golpes de Estado (como en $1948,1960,1961$ y 1979), elecciones no-competitivas (por la presencia de candidatos únicos, como en 1935, 1945 y 1962) o semicompetitivas y fraudes electorales (como los de 1972 y 1977).

Los principales partidos que llevaron a su candidato a la presidencia entre 1935 y 1979 fueron: PRO-PATRIA (Maximiliano Hemández Martínez, 193544), Partido Agrario (Salvador Castaneda Castro, 1945-48), PRUD (Oscar Osorio, 1950-56; José María Lemus, 1956-60) y Partido de Conciliación Nacional (PCN) (Julio Adalberto Rivera, 1962-67; Fidel Sánchez Hernández, 1967-72; Arturo Armando Molina, 1972-77 y Carlos Humberto Romero, 1977-79) . 
El período constitucional para la presidencia durante todo el siglo $\mathrm{XX}$ fluctuó entre cuatro y seis años, y sólo a partir de 1962 se fijó en cinco años, el cual sigue vigente hasta la fecha (Krennerich, 1992). Desde la Constitución de 1886, se exigió la mayoría (absoluta) para la elección del presidente y sólo para 1950 y 1956 se estableció la simple mayoría como requisito para declarar al vencedor. Para las elecciones de 1967 , se preveía otra vez la necesidad de la mayoría absoluta. Sin embargo, conforme a la ley electoral de 1961, si ningún candidato la obtenía, sería el Parlamento quien decidiría entre los dos más votados.

Los principales partidos en la oposición antes de 1982 fueron el PAR (1950 y 1967) y el Partido Demócrata Cristiano (1967, 1972 y 1977). El Cuadro 9 muestra los votos obtenidos por los principales partidos, así como el Número Efectivo de Partidos electorales para los casos en que los datos son confiables. Con base en esta información se puede constatar que cuando existió más de un candidato a la presidencia y se utilizó la fórmula de mayoría absoluta, el formato del sistema de partidos tendía hacia los tres partidos (2.7 en 1967), con una oposición dividida en dos principales partidos (PDC y PAR). Cuando hubo una coalición en la oposición, formando un solo polo electoral (la UNO), el formato se acercaba a 2 y la alternancia en el gobierno podría haber sido posible si no se hubiese recurrido al fraude electoral (1972 y 1977).

\section{Cuadro 9}

Proporción de votos de los principales partidos y/o coaliciones en elecciones presidenciales (1935 y 1979)

\begin{tabular}{l|ccc}
\hline Año & Partido & \% de votos & Observación \\
\hline 1935 & PRO-PATRIA & nd & Candidato único \\
1945 & AGRARIO & 99.7 & Candidato único \\
1956 & PRUD & 56.4 & Nv $=2.0$ \\
& PAR & 43.6 & \\
1956 & PRUD & 95.2 & Nv $=1.1$ \\
1962 & PCN & 100.0 & Candidato único \\
1967 & PCN & 54.4 & Nv $=2.7$ \\
& PDC & 21.6 & \\
1972 & PAR & 14.4 & \\
& PCN & $43.4(?)$ & Fraude electoral y Coalición \\
1977 & UNO & $42.1(?)$ & (PDC-MNR-UDN) \\
& PCN & $67.3(?)$ & Fraude electoral y Coalición \\
& UNO & $32.7(?)$ & (PDC-MNR-UDN) \\
\hline
\end{tabular}

Fuente: Krennerich (1992). 


\subsection{La formación de mayorías entre 1982 y 1994}

La Constitución Política emanada en 1983 estableció la necesidad de una segunda vuelta entre los dos candidatos con más votos, cuando ningún partido alcanzara en la primera vuelta la mayoría absoluta. Esta situación ocurrió tanto en 1984 como en 1994.

En 1984, ninguno de los partidos mayoritarios (Partido Demócrata Cristiano y ARENA) era partido de gobiemo y, por tanto, no puede hablarse de oposición dividida en sentido estricto. El formato del sistema de partidos, a pesar de competir ocho, presentaba un $\mathbf{N}=3.2$. Los principales partidos fueron ARENA, PDC y PCN, que juntos concentraron el 92.8 por ciento de los votos en la primera vuelta. Si se consideran las afinidades entre ARENA y PCN, y las preferencias del gobierno estadounidense hacia el PDC para legitimar su política de contrainsurgencia en el país, se puede decir que la "oposición" al PDC estaba dividida. ARENA y el Partido de Conciliación Nacional (PCN) sumaban juntos el 49.2 por ciento de los votos válidos. Un bloque de derecha que incluyera a PAISA o al PPS hubiese alcanzado la mayoría absoluta. La división de la derecha favoreció el triunfo del PDC, porque en la segunda vuelta la figura del candidato arenero perjudicó sus aspiraciones $\mathrm{y}$, como votando por el mal menor, la mayoría del electorado se inclinó hacia el Partido Demócrata Cristiano (PDC).

En 1989, el Partido Demócrata Cristiano era el partido del gobiemo, pero estaba ya en pleno debilitamiento electoral desde 1988. Por su parte, ARENA había consolidado su "voto duro" alrededor del 29 por ciento desde 1982 y se encontraba en pleno ascenso desde 1988, cuando alcanzó el 48 por ciento de los votos válidos, capturando parte del electorado democristiano que se sentía defraudado por su partido. En 1988, Ns = 2.4, con ARENA y el PDC concentrando el $\mathbf{8 3 . 2}$ por ciento de los votos. En estas condiciones, ARENA triunfa en primera vuelta en las elecciones presidenciales de 1989 con el 53.8 por ciento de los votos. Nv resultó de 2.4. ARENA y el Partido Demócrata Cristiano concentraron $\mathbf{8 9 . 8}$ por ciento de los votos, constituyéndose en los competidores efectivos.

El Nv de 2.4 expresa una situación de enfrentamiento entre dos grandes polos, de acercamiento al bipartidismo. Bajo la mayoría absoluta, esta resultó ser la condición óptima para la alternancia. Una situación que recuerda la planteada en 1972 y 1977, cuando la UNO (coalición opositora) desafío seriamente la continuidad del PCN como partido de gobiemo.

La elección parlamentaria de 1991 incrementó el formato del sistema de partidos. Nv fue de 3.3 y Ns alcanzó el valor de 3. La elección presidencial de 1994 también dio un formato de tres a pesar de los nueve partidos en competencia (tres de ellos en coalición). Como se sabe, los principales partidos de 1994 fueron ARENA, la Coalición FMLN-CD-MNR y el Partido Demócrata Cristia- 
no. Juntos concentraron en primera vuelta el 90.4 por ciento de los votos válidos, pero sólo el 74.1 por ciento correspondía a los dos primeros. De manera análoga a la situación que gozó el PDC en 1984, esta vez ARENA tenía dos polos electorales en la oposición: el PDC y la Coalición. En estas condiciones, como en 1984, y con una concentración de votos para los dos primeros partidos por debajo de los 80 puntos porcentuales, fue necesaria la segunda vuelta y allí, bajo la simple mayoría, volvió a ganar ARENA. Una segunda vuelta realizada prácticamente por puro trámite, dada la enorme ventaja con que el partido de gobiemo superó a su contrincante.

\section{Conclusión}

Este trabajo ha tenido un carácter más descriptivo que explicativo (causal). He pretendido estudiar, con cierto detenimiento, cómo se forman las mayorías en las elecciones presidenciales salvadoreñas tomando en cuenta, por un lado, los niveles de competitividad electoral y márgenes de victoria y, por otro lado, el formato del sistema de partidos. A pesar de que los niveles de competitividad electoral no son muy altos (salvo para el año 1997), el formato del sistema de partidos "empuja" hacia el "ballotage" en presencia de mayoría absoluta. Dicho de otra forma, los niveles de competitividad en las elecciones presidenciales salvadoreñas permiten cuestionar el mecanismo institucional elegido para formar mayorías que legitimen ampliamente al partido ganador, dado que históricamente el partido que ganó la primera vuelta también ganó en la segunda vuelta. Si ésta se vuelve un "mero trámite" que sólo confirma ampliamente al ganador en primera vuelta, lo mejor es bajar el umbral exigido para evitar el ballotage, ya sea por razones financieras o para evitar el desgaste procedimental que conlleva su realización. En El Salvador se ha elegido un diseño que recurrentemente nos lleva a que, para elegir presidente, cada cinco años haya que realizarse dos vueltas electorales. La segunda, para confirmar (legitimar ampliamente) al ganador de la primera. Pregunto: ino es mejor que la legitimidad del ganador, que no su simple confirmación, deba venir más bien por el acatamiento de las "reglas del juego" por todos los competidores antes que por una mayoría absoluta de votos? Lo normado al respecto en el resto de países centroamericanos y los argumentos de este trabajo deberían constituir material de reflexión para los interesados.

\section{Notas}

1. Profesor-Investigador del Departamento de Sociología y Ciencias Políticas de la UCA (El Salvador) y candidato a Doctor en Ciencia Política de la Universidad de Salamanca (España).

2. Otros autores identifican el margen de victoria con la competitividad electoral (Montero, 1992). Yo prefiero hacer distinción entre ambos conceptos, pues si el primero resulta del 10 y 30 por ciento en dos elecciones consecuti- 
vas, lo que intuitivamente sugieren esos valores es que la competitividad ha bajado porque la distancia entre los dos primeros partidos ha aumentado. $\mathrm{Si}$ ambos conceptos fueran idénticos, estaríamos obligados a decir que las elecciones se han vuelto más competitivas, lo cual no parece corresponder con lo que indican aquellos valores.

3. El índice de fraccionalización se calcula con las fórmulas: $\mathrm{F}=1-\sum \mathrm{Vi}^{2}$ o $\mathrm{F}$ $=1-\sum \mathrm{Si}^{2}$, donde $\mathrm{Vi}$ y $\mathrm{Si}$ son las proporciones de votos y escaños del i-ésimo partido.

4. El número efectivo de partidos se calcula mediante la fórmula $\mathrm{Nv}=1 / \sum \mathrm{Vi}^{2}$ o $\mathrm{Ns}=1 / \sum \mathrm{Si}^{2}$, donde $\mathrm{Vi}$ y $\mathrm{Si}$ son las proporciones de votos y escaños del i-ésimo partido. Nv se refiere a los "partidos electorales" y Ns a los "partidos parlamentarios".

5. La relación entre ambos índices está expresada por la expresión $\mathbf{N}=1 /$ ( 1 F).

6. El autor no desarrollará esta argumentación porque no la considera relevante para la temática de este trabajo.

7. Lo fluido del sistema hace referencia a los problemas para estructurar una oferta partidista que sea estable en el tiempo. Para mayores detalles puede consultarse Artiga González (1998).

8. De estos ocho presidentes, tres fueron candidatos únicos (Hemández Martínez, Castaneda Castro y Rivera); dos ganaron por fraude (Molina y Romero) y todos eran militares.

\section{Referencias bibliográficas}

Artiga González, Álvaro, "Fluidez y Volatilidad en la institucionalización de los sistemas de partidos (Notas metodológicas para su medición)", en América Latina Hoy (segunda época), 19, 1998, pp. 125-129.

Duverger, Maurice, Los partidos políticos, México: Fondo de Cultura Económica, 1974.

Krennerich, Michael, "Competitividad de las elecciones en Nicaragua, El Salvador y Guatemala en una perspectiva histórica comparada", en Nohlen, Dieter (ed.), Elecciones y sistemas de partidos en América Latina, San José: IIDH-CAPEL, Costa Rica, 1993, pp. 169-203.

Krennerich, Michael, "El Salvador", en Nohlen, Dieter (comp.), Enciclopedia electoral Latinoamericana y del Caribe, San José: IIDH, Costa Rica, 1992, pp. 307-330.

Laakso, Markku y Taagepera, Rein, "Effective Number of Parties. A measure with Application to West Europe", en Comparative Political Studies, 1, Vol. 12, 1979, pp. 3-27.

Lijphart, Arend, Electoral Systems and Party Systems, New York: Oxford University Press, 1994.

Lijphart, Arend, Las democracias contemporáneas, Barcelona: Ariel, 1987.

Nohlen, Dieter, Sistemas electorales y partidos políticos, México: Fondo de Cultura Económica, 1994.

Rae, Douglas, The political consequences of electoral laws, New Haven: Yale University Press, 1963. 
Rial, Juan, "Partidos políticos, leyes de Duverger y Sartori”, en Revista Uruguaya de Ciencia Política, 2, 1988, pp. 93-108.

Sartori, Giovanni, Partidos y Sistemas de partidos, Madrid: Alianza, 1992. 